\title{
INVARIANT MEANS AND CONES WITH VECTOR INTERIORS
}

\author{
BY \\ R. J. SILVERMAN
}

1. Introduction. The Hahn-Banach theorem on the extension of distributive functionals bounded from above by a positive-homogeneous subadditive functional and the theorem on the extension of monotone distributive functionals can be generalized by taking an ordered linear space with certain special properties as the range space instead of the real number field and requiring that the extension be invariant with respect to certain collections of distributive operators on the domain space. Theorems of this nature have been proved in $[1 ; 6 ; 7 ; 8]$. It will be shown that a semigroup of operators $G$ on the domain space, and the range space $V$ permit invariant Hahn-Banach and monotone extensions if $G$ has an invariant mean and $V$ is a boundedly complete vector lattice whose cone of positive elements is sharp and has a vector interior point.

2. Preliminaries. In this section needed background material is presented. The linear spaces are presumed to have the real numbers as the scalar field. A linear space $V$ is an ordered linear space if there exists a transitive binary relationship $\geqq$ in $V$ such that if $x, y, z$, are in $V$ and $t$ is a nonnegative real number, then if $x \geqq y, t x \geqq t y$, and $x+z \geqq y+z$. The ordering is sharp if $x \geqq y \geqq x$ imply $x=y$. A nonempty subset $C$ of a linear space $V$ is a cone, if $x, y$ are in $C$ and $t$ is a nonnegative real number then $x+y$ and $t x$ are in $C$. A cone $C$ is sharp if $x$ and $-x$ are in $C$ imply $x=0$. A cone $C$ in a linear space $V$ determines an ordering which makes $V$ an ordered linear space. $x \geqq y$ if and only if $x-y$ is in $C$. The set of elements greater than or equal 0 in an ordered linear space is a cone and the ordering determined from this cone is the original ordering. A cone is sharp if and only if its associated ordering is sharp. The definitions of upper bound, lower bound, least upper bound, greatest lower bound and other terms associated with partially ordered systems are assumed.

A function $f$ from an ordered linear space $X$ to an ordered linear space $Y$ is monotone if $x \geqq x^{\prime}$ implies $f(x) \geqq f\left(x^{\prime}\right)$ and $f$ is nonnegative if $x \geqq 0$ implies $f(x) \geqq 0$. If $f$ is a distributive function, the monotone property of $f$ is equivalent to the nonnegative property of $f$. The following theorem is proved in [2].

A. Consider an ordered linear space $V$, then statements 1,2 , and 3 are equivalent and they imply 4 .

1. $V$ is a boundedly complete vector lattice. That is, every set of elements in $V$ with an upper bound has a least upper bound.

Received by the editors October 25, 1956. 
2. Given subsets $A$ and $B$ of $V$ such that $A \geqq B$ (i.e. $a \geqq b, a \in A$ and $b \in B$ ), there exists a $v$ in $V$ such that $A \geqq v \geqq B$.

3. $V$ has the monotone extension property. That is, if (1) $X$ is an ordered subspace of an ordered linear space $Y$ with cone $C$ such that the cone in $X$ is $X \cap C$, (2) for all $y$ in $Y,(y+X) \cap C \neq \phi$ if and only if $(-y+X) \cap C \neq \phi$, then every monotone distributive function from $X$ to $V$ has a monotone distributive extension from $Y$ to $V$.

4. Vhas the Hahn-Banach extension property. That is, if (1) $X$ is a subspace of a linear space $Y,(2) p$ is a positive-homogeneous subadditive function from $Y$ to $V$, (3) $f$ is a distributive function from $X$ to $V$ such that $f(x) \leqq p(x)$, $x \in X$, then there exists a distributive extension $F$ of $f$ from $Y$ to $V$ such that $F(y) \leqq p(y)$ for every $y$ in $Y$.

A representation of a semigroup $\bar{G}$ is defined to be a homomorphism or anti-homomorphism of $\bar{G}$ into the space of distributive operators on a linear space (or the image of $\bar{G}$ under such a map).

Definition 1 . The pair $[G, V]$ where

(a) $\bar{G}$ is an abstract semigroup,

(b) $V$ is a boundedly complete vector lattice whose positive cone $K$ is sharp, has

$(\alpha)$ the Hahn-Banach extension property, if and only if for every collection $[Y, X, G, p, f]$, where (i) $Y$ is a linear space, (ii) $X$ is a subspace of $Y$, (iii) $G$ is a representation of $\bar{G}$ on $Y$ such that $g x \in X$ for $x \in X$ and $g \in G$, (iv) $p$ is a positive-homogeneous subadditive function from $Y$ to $V$ such that $p(g y) \leqq p(y)$ for $g \in G$ and $y \in Y$, and (v) $f$ is a distributive function from $X$ to $V$ such that $f(x) \leqq p(x)$ and $f(g x)=f(x)$ for $y \in Y$ and $g \in G$, there exists a distributive extension $F$ of $f$ to all of $Y$ such that $F(y) \leqq p(y)$ and $F(g y)=F(y)$ for every $y \in Y$ and $g \in G$;

$(\beta)$ the monotone extension property if and only if for every collection $[Y, C, X, G, f]$, where (i) $Y$ is an ordered linear space with positive cone $C$, (ii) $X$ is an ordered linear subspace of $Y$ (with order induced by $C$ ) such that $(y+X) \cap C \neq \phi, y \in Y$, (iii) $G$ is a representation of $\bar{G}$ on $Y$ such that $g z$ is in $C$ and $g x$ is in $X$ for $g$ in $G, z$ in $C$, and $x$ in $X$, and (iv) $f$ is a monotone distributive function from $X$ to $V$ such that $f(g x)=f(x)$ for each $x$ in $X$ and $g$ in $G$, there exists a monotone distributive extension $F$ of $f$ to all of $Y$ such that $F(g y)=F(y)$, for $y$ in $Y$ and $g$ in $G$.

The following theorem is proved in $[7 ; 8]$.

B. The pair $[\bar{G}, V]$ has the monotone extension property if and only if it has the Hahn-Banach extension property.

A semigroup $\bar{G}$ has an invariant mean if there exists a positive linear functional $m$ of unit norm defined over the Banach space of bounded real-valued functions over $\bar{G}, M(\bar{G})$, which is invariant with respect to the right and left regular representations of $\bar{G}$ on $M(\bar{G})$.

For a discussion of semigroups with invariant means see [3]. 
A positive cone $C$ in an ordered linear space $W$ is reproducing if every element in $W$ is the difference of two elements in $C$. If $U$ is a subspace of the space of distributive functionals, $W^{\sharp}$ of an ordered linear space $W$ with cone $C$, then the induced cone in $U, C(U)=\{u \in U \mid u(w) \geqq 0, w \in C\}$ is a cone and determines an ordering so that $U$ is an ordered linear space. The next theorem is proved in [8].

C. Let $W$ be an ordered linear space with reproducing cone. Let $V$ be an ordered linear space with cone $K$, such that $V$ satisfies properties (b) of Definition 1, and in addition is a subspace of the space of distributive functionals of $W$ such that $K$ is the cone induced in $V$ from $W$. Let $\bar{G}$ be a semigroup with an invariant mean. Then $[\bar{G}, V]$ has the monotone extension property and the Hahn-Banach extension property.

3 . Cones with vector interiors. A set $N$ in a linear space $V$ is a vector. neighborhood of $v_{0}$ in $V$ if it contains $v_{0}$ and contains a segment of every line through $v_{0}$ subject to the restriction that $v_{0}$ is not an end point of any of these segments. A set in a linear space has a vector interior if it contains a point along with a vector neighborhood of that point. Every neighborhood of a linear topological space is a vector neighborhood. Further, if $v_{0}$ is a vector interior point of a set $A$ and $v_{0}$ is in a subspace $X$ of $V$ then every translate of $X$ by an element of $V$ meets $A$. The principle theorem of this paper can now be proved.

THEOREM. If $V$ is a boundedly complete vector lattice whose positive cone $K$ is sharp and has a vector interior point $v_{0}$, and if $\bar{G}$ is a semigroup with an invariant mean, then $[\bar{G}, V]$ has the monotone extension property and the HahnBanach extension property.

Proof. Consider $V^{\sharp}$, the space of distributive functionals on $V$. Let $K_{1}=\left\{f\right.$ in $V^{\sharp} \mid f(v) \geqq 0, v$ in $\left.K\right\}$. Then clearly, $V_{1}$, the linear hull of $K_{1}$, is an ordered linear space whose positive cone $K_{1}$ is reproducing. Further, the set $K_{1}$ distinguishes positive elements in $V$ from nonpositive elements in $V$. This will be proved in the following lemma.

Lemma. For any $v_{1} \neq 0$ in $V$, there exists a function $f \in K_{1}$ such that $f\left(v_{1}\right) \neq 0$ and if $v_{1}$ is not in $K, f\left(v_{1}\right)<0$.

Proof. Let $X$ be the subspace of $V$ generated by $v_{1}$ and $v_{0}$. Then for any $v$ in $V, v+X$ meets $K$ since $v_{0}$ is an interior point of $K$. There are three cases to consider.

CASE $1 . v_{1} \geqq 0, v_{1} \neq 0$. Let $k=\sup \left\{t \geqq 0 \mid-t v_{1}+v_{0} \geqq 0\right\}$. The set of elements $\left\{-t v_{1}+v_{0} \geqq 0\right\}$ is not empty since $v_{0}$ is a vector interior point of $K$. The number $k$ exists, for otherwise $-v_{1}+v_{0} / t \geqq 0$ for every positive number $t$ and therefore it follows that $-v_{1}=\inf _{t}\left(-v_{1}+v_{0} / t\right) \geqq 0$, a contradiction of the sharpness of $K$.

Define the distributive functional $h$ on $X: h\left(a v_{0}+b v_{1}\right)=a k+b$. If $a v_{0}+b v_{1}$ 
$\geqq 0$, then, by sharpness, $a, b$ cannot be both less than zero. If $a>0$ and $b>0$, then $a k+b>0$. If $a>0$ and $b<0$, then $-b / a \leqq k$ and hence $a k+b \geqq 0$. If $a<0$ and $b>0$, then $v_{0}+b v_{1} / a \leqq 0$. Therefore, $-b / a \geqq k$ and $a k+b \geqq 0$.

CASE 2. $v_{1} \leqq 0, v_{1} \neq 0$. Consider $-v_{1}$ and use Case 1 . Note that Cases 1 and 2 include $v_{1}=s v_{0}$.

CASE 3. $v_{1} \ddagger 0, v_{1} \geq 0$. Let $k=\sup \left\{|t| \mid t v_{1}+v_{0} \geqq 0\right\}$ the number $k$ exists. If for all positive $t, t v_{1}+v_{0} \geqq 0$, then $v_{1}=\inf _{t}\left(v_{1}+v_{0} / t\right) \geqq 0$. Therefore, $t$ is bounded from above. Similarly, it follows that $t$ is bounded from below. Thus $k$ is defined. Define the distributive functional $h$ on $X: h\left(a v_{0}+b v_{1}\right)=a k-b$. Therefore $h\left(v_{0}\right)=k$ and $h\left(v_{1}\right)=-1$. Consider $a v_{0}+b v_{1} \geqq 0$. First, $a \geqq 0$; for otherwise $b v_{1} \geqq-a v_{0} \geqq 0$ and $v_{1}$ is comparable with zero. Since $a \geqq 0, v_{0}+b v_{1} / a$ $\geqq 0$ and $|b| / a \leqq k$. Therefore, $a k-b \geqq a k-|b| \geqq 0$.

Therefore, since the real numbers form a boundedly complete vector lattice, $V, X, K, h$ satisfy the conditions for $Y, X, C$, and $f$ of Theorem A.3, there exists a monotone distributive extension of $h$ to all of $V$, and the lemma is proved.

Consider now $V_{2}$ the linear space of distributive functionals of $V_{1}$. Consider the cone induced in $V_{2}$ from $V_{1}: K_{2}=\left\{w\right.$ in $V_{2} \mid w(f) \geqq 0, f$ in $\left.K_{1}\right\}$. Thus $V_{2}$ is an ordered linear space relative to $K_{2}$. Consider the natural mapping $Q$ from $V$ into $V_{2}: Q v(f)=f(v), v$ in $V, f$ in $V_{1}$. The mapping $Q$ is distributive and monotone clearly. Since $V_{1}$ has sufficiently many functionals $Q$ is $1-1$ onto $Q V \subset V_{2}$. The mapping $Q^{-1}$ from $Q V$ to $V$ is thus defined and in addition, distributive. Further $Q^{-1}$ is monotone, for if $Q v=w$ is in $K_{2}$ then $w(f) \geqq 0$, for $f$ in $K_{1}$. By virtue of the lemma this implies that $v$ is in $K$.

The image of $V$ under $Q, Q V$, is a boundedly complete vector lattice. This follows from Theorem A.2 and the fact that $Q$ and $Q^{-1}$ are monotone mappings.

Consider now $[Y, C, X, G, f]$ as in Definition $1(\beta)$, where $G$ is a representation of $\bar{G}$. Define $f_{1}$ from $X$ to $Q V: Q f=f_{1}$, then the collection $\left[Y, C, X, G, f_{1}\right.$ ] satisfies the conditions in Definition $1(\beta)$. It is now noted that the pair $[\bar{G}, Q V]$, satisfy the conditions of Theorem $\mathrm{C}$. Hence this pair has the monotone extension property. Thus there exists a monotone distributive extension $F_{1}$ of $f_{1}$ to all of $Y$ with values of $Q V$, such that $F_{1}(g y)=F_{1}(y)$ for $y$ in $Y$ and $g$ in $G$. Define $F=Q^{-1} F_{1}$. This is clearly an invariant, monotone, distributive extension of $f$. Hence $[\bar{G}, V]$ has the monotone and the Hahn-Banach extension properties.

A theorem is proved in [8] which implies the converse of the previous theorem and is quoted. If $V$ is a boundedly complete vector lattice with a sharp positive cone with a vector interior point, then, if (1) $Y$ is an ordered linear space with cone $C$, (2) $X$ is a subspace of $Y$ with order induced by $C$, and such that $y+X$ meets $C$ for all $y$ in $Y$, (3) $f$ is a monotone distributive function from $X$ to $V$, (4) $G$ is a semigroup of operators on $Y$ such that $g x$ is in $X, g z$ is in $C$, and $f(g x)=f(x)$, for all $g$ in $G, x$ in $X$, and $z$ in $C$, implies 
that there exists a monotone, distributive extension $F$ of $f$ to all of $Y$ such that $F(g y)=F(y)$, for $g$ in $G$ and $y$ in $Y$, it follows that the semigroup of operators $G$, considered as an abstract semigroup, has an invariant mean.

\section{BIBLIOGRAPHY}

1. S. Banach, Théorie des opérations linéaires, Warsaw, 1932.

2. M. M. Day, Notes on ordered linear spaces, University of Illinois, 1950, unpublished.

3. - Means for the bounded functions and ergodicity of the bounded representations of semigroups, Trans. Amer. Math. Soc. vol. 69 (1950) pp. 89-108.

4. S. Kakutani, Concrete representations of abstract (M)-spaces, Ann. of Math. vol. 42 (1941) pp. 523-537.

5. M. G. KreY̌n and M. A. Rutman, Linear operators leaving invariant a cone in a Banach space [Uspehi Matematicheskii Nauk (N.S.) vol. 3 (23) (1948) pp. 3-95] Amer. Math. Soc. Translations, no. 26, 1950.

6. A. Morse and R. Agnew, Extensions of linear functionals, with applications to limits, integrals, measures, and densities, Ann. of Math. vol. 39 (1938) pp. 20-30.

7. R. J. Silverman, Invariant linear functions, Trans. Amer. Math. Soc. vol. 81 (1956) pp. $411-424$.

8. - - Means on semigroups and the Hahn-Banach extension property, Trans. Amer. Math. Soc. vol. 83 (1956) pp. 222-237.

Illinois Institute of Technology, Chicago, Ill. 